\title{
Mechanisms of HIV Suppression by Various Microbes in Human Lymphoid Tissue Leonid Margolis*‡
}

\author{
Address: Section of Intercellular Interactions, National Institute of Child Health and Human Development, Bethesda, MD 20892 \\ Email: Leonid Margolis* - margolis@helix.nih.gov \\ * Corresponding author ¥Presenting author
}

from 2005 International Meeting of The Institute of Human Virology

Baltimore, USA, 29 August - 2 September 2005

Published: 8 December 2005

Retrovirology 2005, 2(Suppl I):S8 doi: I0. I 186/I 742-4690-2-SI-S8

Various pathogens enhance HIV replication and disease progression in coinfected individuals. However, we, and others, have provided examples of microbial interactions that suppress HIV-1 infection both in vivo and ex vivo. Here, we report on the mechanisms of interactions of HIV-1 with several such microbes in the context of ex vivo infected human lymphoid tissue. Blocks of human tonsils maintained ex vivo were coinfected with R5 or X4 HIV-1 and with another microbe, measles virus (MV), vaccinia, herpesvirus 6 (HHV-6), herpesvirus 7 (HHV-7), cytomegalovirus (CMV), or a parasite, Toxoplasma gondii (TG).

In ex vivo tissues, all the above-listed microbes, except CMV, inhibited replication of R5 HIV-1 whereas replication of X4 HIV-1 was affected mildly. In spite of similarity of the effects, the mechanisms of R5 inhibition by coinfecting microbes are strikingly diverse. HHV-6 and MV upregulate CC-chemokines, in particular RANTES to the levels sufficient to inhibit replication of R5 HIV-1; Toxoplasma gondii seems to secret its own soluble factor, cyclophyline, that also binds to CCR5 coreceptor, HHV-7 downregulates the expression of $\mathrm{CD} 4$ on T cells.

In conclusion, soluble factors encoded by microbes, including their components, or secreted by infected and bystander cells in response to microbial invasion (in particular cytokines/chemokines) constitute a universal network through which microbes interact with human body creating a microenvironment that is beneficial for them. However, what is beneficial for one microbe can be detrimental for another. Deciphering the molecular mechanisms by which pathogens alter tissue microenvironment so that it becomes detrimental to HIV, may significantly contribute to the development of efficient anti-HIV therapies. 